Surgery for Tennis Elbow: a Systematic Review

Keywords: tennis, elbow, surgery, systematic review 


\title{
Surgery for Tennis Elbow: a Systematic Review
}

\author{
M Bateman $^{1}$, C Littlewood $^{2}$, B Rawson $^{3}$, AA Tambe $^{1}$ \\ ${ }^{1}$ Orthopaedic Department, Derby Teaching Hospitals NHS Foundation Trust, Derby, DE22 \\ 3NE,UK. \\ ${ }^{2}$ Arthritis Research UK Primary Care Centre, Research Institute for Primary Care and Health \\ Sciences and Keele Clinical Trials Unit, David Weatherall Building, Keele University, \\ Staffordshire, ST5 5BG, UK. \\ ${ }^{3}$ Library Services, Derby Teaching Hospitals NHS Foundation Trust, Derby, DE22 3NE,UK.
}

\author{
Contact details: \\ Marcus Bateman \\ Orthopaedic Outpatient Department \\ Royal Derby Hospital \\ Derby, DE22 3NE, UK. \\ Email: marcus.bateman@nhs.net \\ Twitter: @marcusbatemanPT
}

Funding: No funding required

Category: Systematic review 


\begin{abstract}
:
Background: There is no consensus on the most suitable treatment for tennis elbow but in the USA surgical intervention is increasing despite a lack of supportive research evidence. The aim of this systematic review was to provide a balanced update based on all relevant published RCTs to date.

Methods An electronic search of MEDLINE, EMBASE, CINAHL, BNI, AMED, PsycINFO, HBE, HMIC, PubMed, TRIP, Dynamed Plus and The Cochrane Library, was complemented by hand searching. Risk of bias was assessed using the Cochrane Risk of Bias Tool and data was synthesised narratively, based on levels of evidence, due to heterogeneity.
\end{abstract}

Results: 12 studies of poor methodological quality were included. The available data suggest that surgical interventions for tennis elbow are not more effective than non-surgical and sham interventions. Surgical technique modifications may enhance effectiveness compared with traditional methods but have not been tested against placebo.

Conclusions: Current research evidence suggests surgery for tennis elbow is no more effective than non-surgical treatment based on evidence with significant methodological limitations. Given the recalcitrant nature of tennis elbow for some patients, further research in the form of a high quality placebo-controlled surgical trial with additional conservative arm, is required to usefully inform clinical practice.

Keywords: tennis, elbow, surgery, systematic review 


\section{INTRODUCTION:}

Tennis elbow is the most common cause of lateral elbow pain and has been reported to have a point prevalence of 1 to $3 \% .{ }^{1}$ Tennis elbow is characterised by pain near the lateral epicondyle that is aggravated by contraction of the extensor muscles, particularly when gripping an object. It commonly affects adults of working age and can affect the individual's ability to work and engage with other activities, including sport. ${ }^{2,3}$ A study of worker's compensation claims in Washington State between 1990 and 1998 found the average work sickness absence for elbow epicondylitis of 219 days with an average claim cost of 8099 US dollars. ${ }^{4}$ There is currently no established consensus on the most appropriate form of treatment for this condition although a treatment algorithm has been proposed but has not yet been evaluated. ${ }^{5}$ Many treatment options are available ranging from conservative measures such as physiotherapy, including exercise, manual therapy and strapping, injections of various substances, including corticosteroid and platelet rich plasma, and surgical debridement. Evidence suggests that although corticosteroid injections might offer short term pain relief, in the long term they lead to worse outcome than a wait-and-see approach and also negate the beneficial effects of therapeutic exercise treatments. ${ }^{5}$

Given the recalcitrant nature of tennis elbow for some patients, surgical intervention might be offered to this group. An increasing trend towards surgery is apparent in the USA with a rise in the proportion of patients with tennis elbow undergoing surgery from $1.1 \%$ in 2000 2002 to $3.2 \%$ in 2009-2011 at the Mayo Clinic (Rochester, USA). ${ }^{6}$ Buchbinder et al ${ }^{7}$ conducted a systematic review of surgery for lateral elbow pain (that included tennis elbow) in 2002 and updated their review in $2011 .^{8}$ The conclusions from both reviews were similar, describing the lack of evidence to support or refute surgery for this condition. In particular there was a lack of high quality randomised controlled trials (RCTs) and specifically none that compared surgery to a placebo intervention. Given the rising incidence of this surgery in the USA and in light of the findings of previous systematic reviews, the aim of this current systematic review was to provide updated guidance based on all relevant published RCTs to date.

\section{METHODS:}

A systematic review was conducted using a predetermined protocol registered on the PROSPERO database of systematic reviews (accessible via www.crd.york.ac.uk/PROSPERO/display_record.asp?ID=CRD42016050849) in accordance with the PRISMA-P statement. ${ }^{9}$ 


\section{Search Strategy:}

An electronic search of MEDLINE, EMBASE, CINAHL, BNI, AMED, PsycINFO, HBE, HMIC, PubMed, TRIP database, The Cochrane Library, Dynamed Plus, NICE Guidance, CKS, SIGN and Specialist websites: including RGN, NIHR(National Institute for Health Research) was conducted by a medical librarian (BR) on March $8^{\text {th }} \& 9^{\text {th }} 2017$ using search terms detailed in Figure 1. An example search of the MEDLINE database is shown in Figure 2. The database searches were supplemented by hand searches of abstracts presented at the British Elbow \& Shoulder Society annual scientific meeting 2016 as these had yet to be published in the UK journal of Shoulder \& Elbow and therefore yet to be indexed on PubMed.

Databases searched:

- Evidence Based Reviews: The Cochrane Library

- Guidance: Dynamed Plus, NICE Guidance, CKS, SIGN,

- Healthcare Databases: MEDLINE, EMBASE, CINAHL, BNI, AMED, PsycINFO, HBE, HMIC, PubMed, TRIP database,

- Specialist websites: e.g. RGN, NIHR(National Institute for Health Research),

Search Terms:

\begin{tabular}{|l|l|}
\hline MeSH Headings & Free Text Words \\
\hline exp TENNIS ELBOW & ("lateral epicondylitis") \\
\hline exp SURGICAL & ("lateral epicondylosis") \\
PROCEDURES, OPERATIVE & \\
\hline exp DEBRIDEMENT/ & ("lateral epicondylalgia") \\
\hline $\begin{array}{l}\text { exp DOCUMENT TYPE } \\
\text { RANDOMIZED CONTROLLED } \\
\text { TRIALS/ }\end{array}$ & (surgical) \\
\hline & (debridement) \\
\hline & (release) \\
\hline & ("randomised controlled trial') \\
\hline & ("randomized controlled trial") \\
\hline & (RCT) \\
\hline
\end{tabular}

Search Limits:

Randomised Controlled Trials

[Figure 1: Search Strategy] 
1 Medline exp "TENNIS ELBOW"/

2 Medline

("lateral epicondylitis").ti,ab

726

3 Medline

("lateral epicondylosis").ti,ab

44

$4 \quad$ Medline

("lateral epicondylalgia").ti,ab

121

$5 \quad$ Medline

(1 OR 2 OR 3 OR 4)

1745

6 Medline

exp "SURGICAL PROCEDURES, OPERATIVE"/

2741554

$7 \quad$ Medline

(surgical).ti,ab

792860

$8 \quad$ Medline

(debridement).ti,ab

19780

$9 \quad$ Medline

exp DEBRIDEMENT/

13741

10 Medline

(8 OR 9)

26625

11 Medline

(release).ti,ab

486574

12 Medline

(6 OR 7 OR 10 OR 11)

3596659

13 Medline

(5 AND 12)

536

14 Medline

13 [Document type Randomized Controlled Trial]

[Figure 2: Example search strategy using Medline database]

Two reviewers ( $M B$ \& $C L$ ) then independently screened titles and abstract before selecting full-text papers, where available, based on pre-defined inclusion criteria:

- Adults diagnosed with Tennis Elbow

- Any form of surgical intervention 
- Any form of comparator treatment including other forms of surgery, injections, physiotherapy, sham surgery or wait-and-see.

- Randomised controlled trials only

- English language

- Primary outcomes: Patient reported outcome measures of pain and function

- Secondary outcomes: Return to work, return to sport

A third reviewer (AT) was available for arbitration in the event of disagreement but was not required. The study selection process is detailed in Figure 3.

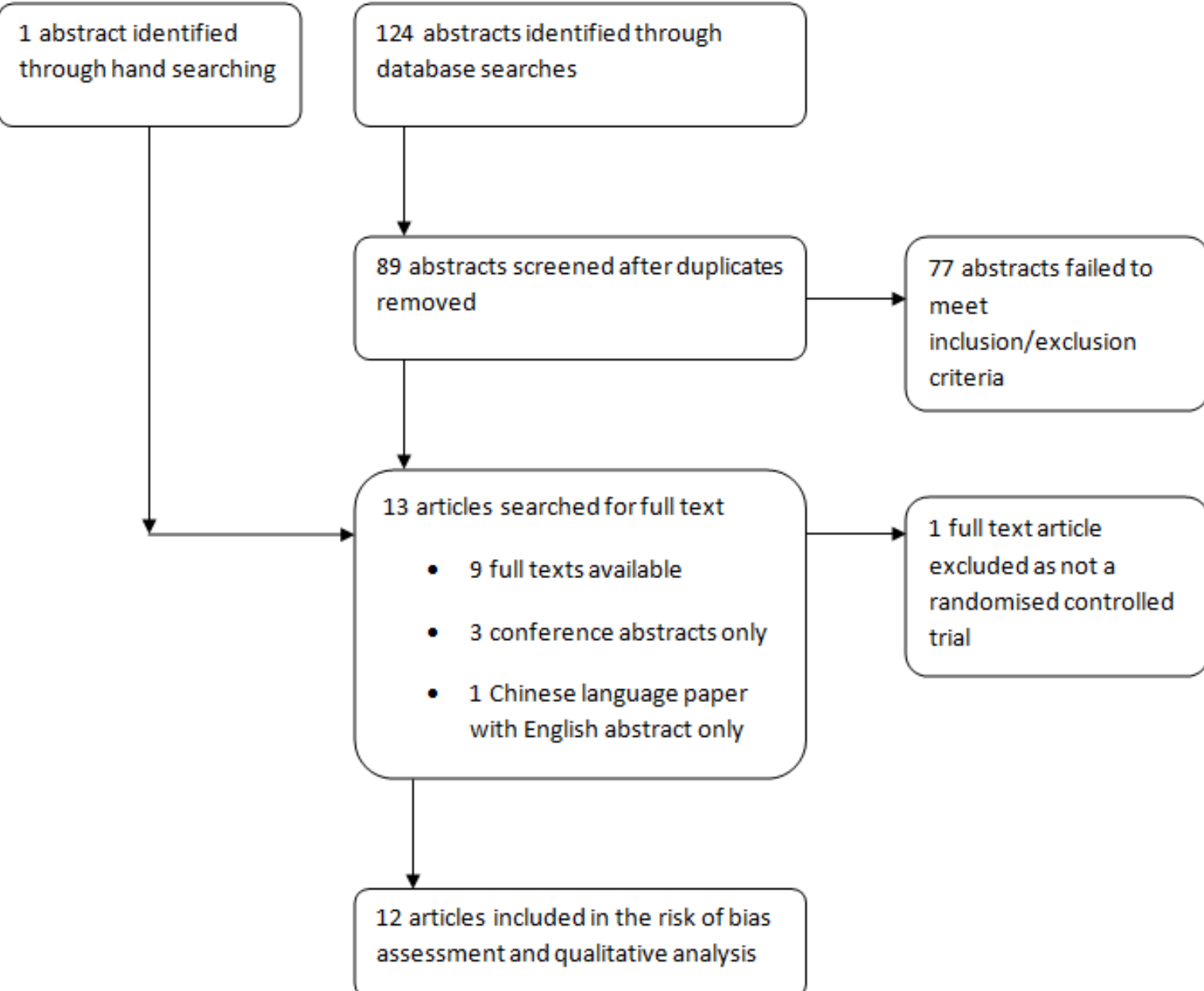

[Figure 3: Study Selection Flowchart] 


\section{Data extraction:}

One reviewer (MB) extracted data in relation to study characteristics, participant characteristics, interventions and results before a second reviewer $(C L)$ independently verified the findings. The extracted data is presented in Table 1.

\begin{tabular}{|c|c|c|c|}
\hline Study Characteristics & $\begin{array}{l}\text { Participant } \\
\text { Characteristics }\end{array}$ & Interventions & Results \\
\hline $\begin{array}{l}\text { Dunkow et al } 2004^{10} \\
\text { RCT comparing open } \\
\text { Nirschl release }{ }^{23} \text { vs } \\
\text { percutaneous tenotomy } \\
\text { Conducted in the UK }\end{array}$ & $\begin{array}{l}45 \text { patients ( } 47 \\
\text { elbows) } \\
\text { Age range } 30-58 \\
22 \text { male, } 25 \text { female } \\
\text { Failed } 12 \text { months of } \\
\text { conservative } \\
\text { treatment including } \\
\text { two } 80 \mathrm{mg} \\
\text { hydrocortisone } \\
\text { injections }\end{array}$ & $\begin{array}{l}\text { Open Nirschl release } \\
\text { with } 3 \text { drill holes into } \\
\text { the lateral epicondyle } \\
(n=24) \\
\text { Percutaneous ( } 1 \mathrm{~cm} \\
\text { incision) division of the } \\
\text { common extensor } \\
\text { origin ( } n=23 \text { ) } \\
\text { Standardised } \\
\text { physiotherapy for both } \\
\text { groups post- } \\
\text { operatively }\end{array}$ & $\begin{array}{l}\text { 12-month follow up: } \\
\text { Significant } \\
\text { improvements in } \\
\text { Disability of Arm } \\
\text { Shoulder Hand (DASH) } \\
\text { score ( } p=0.001 \text { ) in both } \\
\text { groups } \\
\text { Patient satisfaction in } \\
\text { favour of percutaneous } \\
\text { group ( } p=0.012 \text { ) } \\
\text { Median return to work } \\
5 \text { weeks in open group, } \\
2 \text { weeks in } \\
\text { percutaneous group. } \\
P=0.0001 \text { in favour of } \\
\text { percutaneous group }\end{array}$ \\
\hline $\begin{array}{l}\text { Keizer et al } 2002^{11} \\
\text { RCT pilot study } \\
\text { comparing botulinum } \\
\text { toxin (botox) injection vs } \\
\text { open release (Hohmann } \\
\text { method }{ }^{24} \text { ) } \\
\text { Conducted in The } \\
\text { Netherlands }\end{array}$ & $\begin{array}{l}40 \text { patients } \\
\text { Mean age } 42.8 \text { (25- } \\
72 \text { ) } \\
19 \text { male, } 21 \text { female } \\
\text { Mean duration of } \\
\text { symptoms } 10.5 \\
\text { months with } \\
\text { minimum of } 6 \\
\text { months. } \\
\text { Failed conservative } \\
\text { treatment } \\
36 \text { patients had } \\
\text { previous steroid }\end{array}$ & $\begin{array}{l}30-40 \text { units of botox } \\
\text { injected into the } \\
\text { extensor carpi radialis } \\
\text { brevis (ECRB) ( } n=20) .8 \\
\text { patients given a } \\
\text { second injection due to } \\
\text { limited effect. } \\
\text { Hohmann technique of } \\
\text { open release }{ }^{24} \text { of ECRB } \\
\text { ( } n=20 \text { ) followed by } \\
\text { sling for } 2 \text { weeks }\end{array}$ & $\begin{array}{l}\text { Outcomes measured } \\
\text { by modified Verhaar } \\
\text { score }{ }^{25} \text { : } \\
4 \text { patients in the botox } \\
\text { group underwent open } \\
\text { surgery due to failed } \\
\text { response. Outcomes } \\
\text { for these patients at } 24 \\
\text { months were } 1 \text { good, } 1 \\
\text { fair, } 2 \text { poor. } \\
\text { Main results at } 24 \\
\text { months: } \\
\text { Botox: } 11 / 16 \text { excellent } \\
\text { result, } 4 / 16 \text { good, } 1 / 16\end{array}$ \\
\hline
\end{tabular}




\begin{tabular}{|c|c|c|c|}
\hline & injections & & $\begin{array}{l}\text { fair. } \\
\text { Open surgery: } 14 / 20 \\
\text { excellent result, } 3 / 20 \\
\text { good, } 3 / 20 \text { poor. } \\
\text { Overall no significant } \\
\text { differences reported in } \\
\text { pain or range of } \\
\text { motion at } 3,6,12 \text { or } \\
24 \text { months. } \\
\text { Sick leave in favour of } \\
\text { surgery group at } 3 \\
\text { months ( } p=0.01 \text { ) but } \\
\text { no difference at } 6,12 \text {, } \\
24 \text { months }\end{array}$ \\
\hline $\begin{array}{l}\text { Khashaba } 2001^{12} \\
\text { RCT comparing Nirschl } \\
\text { technique of open } \\
\text { release }^{23} \text { with or without } \\
\text { drilling the bone of the } \\
\text { anterolateral humeral } \\
\text { condyle } \\
\text { Conducted in the UK }\end{array}$ & $\begin{array}{l}18 \text { patients ( } 23 \\
\text { elbows) } \\
\text { Aged over } 18 \\
\text { Failed to improve } \\
\text { with rest and } \\
\text { physiotherapy } \\
\text { Temporary (<6 } \\
\text { months) response to } \\
\text { steroid injection } \\
40 \text { mg depo- } \\
\text { medrone }\end{array}$ & $\begin{array}{l}\text { Nirschl open release }{ }^{23} \\
\text { including } 3 \text { drill holes } \\
\text { into the lateral } \\
\text { epicondyle ( } n=9 \\
\text { patients but number of } \\
\text { elbows not stated) } \\
\text { Nirschl open release }{ }^{23} \\
\text { without the drilling } \\
\text { component ( } n=9 \text { but } \\
\text { number of elbows not } \\
\text { stated) }\end{array}$ & $\begin{array}{l}\text { Mean wrist extension } \\
\text { power improvement } \\
\text { using AK-7000 } \\
\text { extensionometer at } 6 \\
\text { months: drilled } 5.2 \mathrm{~kg} \text {, } \\
\text { non-drilled } 6.5 \mathrm{~kg} \\
\text { Mean improvement in } \\
\text { pain Visual Analogue } \\
\text { Scale (VAS) } 3-6 \\
\text { months: drilled } 4.6 \mathrm{~cm} \text {, } \\
\text { non-drilled } 6.8 \mathrm{~cm}\end{array}$ \\
\hline $\begin{array}{l}\text { Kroslak \& Murrell } 2017^{13} \\
\text { RCT comparing open } \\
\text { Nirschl release }{ }^{23} \text { vs sham } \\
\text { surgery (skin incision } \\
\text { only) } \\
\text { Conducted in Australia } \\
\text { Conference abstract only }\end{array}$ & $\begin{array}{l}26 \text { patients } \\
\text { Groups matched for } \\
\text { age, gender, hand } \\
\text { dominance, duration } \\
\text { of symptoms } \\
\text { Chronic tennis elbow } \\
\text { for minimum } 6 \\
\text { months } \\
\text { Failed two non- } \\
\text { surgical modalities }\end{array}$ & $\begin{array}{l}\text { Nirschl open release } \\
(n=13) \\
\text { Sham surgery involving } \\
\text { skin excision and } \\
\text { exposure to level of } \\
\text { ECRB tendon }(n=13)\end{array}$ & $\begin{array}{l}\text { Both groups improved } \\
\text { subjective outcomes at } \\
6 \text { and } 12 \text { months } \\
(p<0.01) . \\
\text { Both groups improved } \\
\text { tenderness, } \\
\text { pronation/supination } \\
\text { range, grip strength } \\
\text { and ORI-TETS score }{ }^{26} \\
\text { at } 6 \text { months ( } p<0.05) \text {. } \\
\text { No differences } \\
\text { between groups in any } \\
\text { measure at any time }\end{array}$ \\
\hline
\end{tabular}




\begin{tabular}{|c|c|c|c|}
\hline & & & $\begin{array}{l}\text { point. } \\
\text { Study stopped early } \\
\text { due to lack of } \\
\text { difference between } \\
\text { groups. }\end{array}$ \\
\hline $\begin{array}{l}\text { Leiter et al } 2016^{14} \\
\text { RCT comparing open vs } \\
\text { arthroscopic release } \\
\text { Conducted in Canada } \\
\text { Conference abstract only }\end{array}$ & $\begin{array}{l}71 \text { patients } \\
\text { Aged over } 16 \\
\text { Open group: mean } \\
\text { age } 47.1 ; 19 \text { male, } \\
15 \text { female } \\
\text { Arthroscopic group: } \\
\text { mean age } 45.0 ; 21 \\
\text { male, } 13 \text { female } \\
\text { Failed to improve } \\
\text { with } 6 \text { months } \\
\text { conservative } \\
\text { treatment }\end{array}$ & $\begin{array}{l}\text { Open tennis elbow } \\
\text { release }(n=34) \\
\text { Arthroscopic tennis } \\
\text { elbow release }(n=34) \\
\text { ? Some individuals had } \\
\text { bilateral surgery due to } \\
\text { discrepancy in total } \\
\text { number of patients }\end{array}$ & $\begin{array}{l}\text { No difference in pain } \\
\text { VAS, DASH score or } \\
\text { grip power between } \\
\text { groups at any time } \\
\text { point up to } 12 \text { months. } \\
\text { 17/34 met the } \\
\text { minimally clinically } \\
\text { important difference } \\
\text { (MCID) in DASH score } \\
\text { in the open group and } \\
\text { 19/34 in the } \\
\text { arthroscopic group. }\end{array}$ \\
\hline $\begin{array}{l}\text { Leppilahti et al } 2001^{15} \\
\text { RCT comparing open } \\
\text { posterior interosseous } \\
\text { nerve decompression vs } \\
\text { open extensor carpi } \\
\text { radialis brevis tenotomy } \\
\text { Conducted in Finland }\end{array}$ & $\begin{array}{l}26 \text { patients ( } 28 \\
\text { elbows) } \\
\text { Nerve group: mean } \\
\text { age } 42 \text { (33-50); } 6 \\
\text { male, } 7 \text { female } \\
\text { Tenotomy group: } \\
\text { mean age } 41 \text { (30- } \\
52) ; 7 \text { male, } 7 \text { female } \\
\text { Mean symptom } \\
\text { duration } 23 \text { months } \\
\text { (5-60) } \\
\text { Failed conservative } \\
\text { care including } \\
\text { physiotherapy and } \\
\text { mean number of } \\
\text { steroid injections } 4.5\end{array}$ & $\begin{array}{l}\text { Open decompression } \\
\text { of the posterior } \\
\text { interosseous nerve } \\
(n=14) \\
\text { Open z-shaped } \\
\text { tenotomy of the ECRB } \\
(n=14)\end{array}$ & $\begin{array}{l}\text { Mean follow up } 31 \\
\text { months (22-48). } \\
\text { No significant } \\
\text { difference in grip } \\
\text { strength between } \\
\text { groups. } \\
\text { Subjective pain relief: } \\
\text { Nerve group: } 3 \\
\text { excellent, } 4 \text { good, } 2 \\
\text { fair, } 5 \text { poor. } \\
\text { Tenotomy group: } 2 \\
\text { excellent, } 4 \text { good, } 5 \\
\text { fair, } 3 \text { poor. } \\
\text { Patients undergoing } \\
\text { repeat surgery due to } \\
\text { poor outcome: nerve } \\
\text { group } 4 \text {, tenotomy } \\
\text { group } 3 \text {. }\end{array}$ \\
\hline
\end{tabular}




\begin{tabular}{|c|c|c|c|}
\hline $\begin{array}{l}\text { Meknas et al } 2008^{16} \\
\text { RCT comparing } \\
\text { radiofrequency } \\
\text { microtenotomy vs open } \\
\text { surgical release \& repair } \\
\text { Conducted in Norway }\end{array}$ & $\begin{array}{l}24 \text { patients } \\
13 \text { male, } 11 \text { female } \\
\text { Open surgery: mean } \\
\text { age } 49.2 \text { (36-62). } \\
\text { Mean symptom } \\
\text { duration } 27.6 \\
\text { months } \\
\text { Microtenotomy: } \\
\text { mean age } 46.7 \text { (30- } \\
64) \text {. Mean symptom } \\
\text { duration } 22 \text { months } \\
\text { All patients had } \\
\text { minimum symptoms } \\
12 \text { months with } \\
\text { failed conservative } \\
\text { treatment including } \\
3 \text { months of } \\
\text { physiotherapy and } \\
\text { at least } 3 \text { steroid } \\
\text { injections }\end{array}$ & $\begin{array}{l}\text { Open surgical release } \\
\text { using modified Nirschl } \\
\text { technique }{ }^{23}(n=11) \\
\text { Open } 3 \mathrm{~cm} \text { exposure of } \\
\text { extensor tendon with } \\
\text { microtenotomy using a } \\
\text { radiofrequency device } \\
\text { to a depth of 3-5mm in } \\
3-6 \text { areas }(n=13)\end{array}$ & $\begin{array}{l}\text { No difference in pain } \\
\text { VAS between groups at } \\
3,6,12 \text { weeks or } 10-18 \\
\text { months. } \\
\text { Both groups had } \\
\text { significant pain } \\
\text { reduction from } 6 \\
\text { weeks onward ( } p<0.04 \\
\text { open, } p<0.001 \\
\text { microtenotomy) } \\
23 / 24 \text { patients had } \\
\text { reduced pain at } 10-18 \\
\text { months ( }<0.05 \text { ) } \\
\text { No difference in grip } \\
\text { strength between } \\
\text { groups at } 12 \text { weeks } \\
\text { and no difference to } \\
\text { contralateral side. } \\
\text { No difference in Mayo } \\
\text { Elbow Performance } \\
\text { Score (MEPS) between } \\
\text { groups at } 12 \text { weeks but } \\
\text { significantly better } \\
\text { than pre-op ( } p<0.01 \\
\text { open, } p<0.001 \\
\text { microtenotomy) }\end{array}$ \\
\hline $\begin{array}{l}\text { Meknas et al } 2013^{17} \\
\text { RCT comparing } \\
\text { radiofrequency } \\
\text { microtenotomy vs open } \\
\text { surgical release \& repair } \\
\text { Conducted in Norway }\end{array}$ & $\begin{array}{l}24 \text { patients } \\
13 \text { male, } 11 \text { female } \\
\text { Open surgery: mean } \\
\text { age } 49.2 \text { (36-62). } \\
\text { Mean symptom } \\
\text { duration } 27.6 \\
\text { months } \\
\text { Microtenotomy: } \\
\text { mean age } 46.7 \text { ( } 30- \\
64) \text {. Mean symptom } \\
\text { duration } 22 \text { months }\end{array}$ & $\begin{array}{l}\text { Open surgical release } \\
\text { using modified Nirschl } \\
\text { technique }{ }^{23}(n=11) \\
\text { Open } 3 \mathrm{~cm} \text { exposure of } \\
\text { extensor tendon with } \\
\text { microtenotomy using a } \\
\text { radiofrequency device } \\
\text { to a depth of 3-5mm in } \\
3-6 \text { areas }(n=13)\end{array}$ & $\begin{array}{l}\text { 5-7 year follow up: } \\
1 \text { patient had died due } \\
\text { to unrelated causes. } \\
1 \text { patient in the } \\
\text { microtenotomy group } \\
\text { had revision open } \\
\text { surgery. } \\
\text { Improved pain VAS in } \\
\text { both groups ( } p<0.005 \text { ) } \\
\text { but no differences } \\
\text { between groups. }\end{array}$ \\
\hline
\end{tabular}




\begin{tabular}{|c|c|c|c|}
\hline & $\begin{array}{l}\text { All patients had } \\
\text { minimum symptoms } \\
12 \text { months with } \\
\text { failed conservative } \\
\text { treatment including } \\
3 \text { months of } \\
\text { physiotherapy and } \\
\text { at least } 3 \text { steroid } \\
\text { injections }\end{array}$ & & $\begin{array}{l}\text { No difference in grip } \\
\text { strength between } \\
\text { groups. } \\
\text { Improved MEPS in } \\
\text { both groups ( } p<0.01 \text { ) } \\
\text { but no difference } \\
\text { between groups. }\end{array}$ \\
\hline $\begin{array}{l}\text { Monto } 2014^{18} \\
\text { RCT comparing Nirschl } \\
\text { open release }{ }^{23} \text { and } \\
\text { repair with or without } \\
\text { suture anchors } \\
\text { Conducted in the USA }\end{array}$ & $\begin{array}{l}60 \text { patients with } \\
\text { positive MRI findings } \\
\text { of Tennis Elbow. } \\
\text { No anchor group: } \\
\text { mean age } 48.2 \text { (30- } \\
61 \text { ); } 16 \text { male, } 14 \\
\text { female. } \\
\text { Anchor group: mean } \\
\text { age } 49.3 \text { (30-62); } 18 \\
\text { male, } 12 \text { female. } \\
\text { Failed } 6 \text { months of } \\
\text { conservative } \\
\text { treatment including } \\
\text { minimum } 6 \text { weeks } \\
\text { physiotherapy and } \\
\text { 1-3 steroid } \\
\text { injections. } \\
\text { Mean symptoms } \\
\text { duration: } \\
\text { Anchor group } 10.4 \\
\text { months } \\
\text { No anchor group } 8.9 \\
\text { months }\end{array}$ & $\begin{array}{l}\text { Elbow arthroscopy and } \\
\text { debridement followed } \\
\text { by Nirschl open } \\
\text { release }^{23}(n=30) \\
\text { Elbow arthroscopy } \\
\text { followed by open } \\
\text { debridement, } \\
\text { decortication and } \\
\text { suture reattachment of } \\
\text { ECRB tendon to the } \\
\text { lateral epicondyle } \\
\text { using two polyether } \\
\text { ether ketone (PEEK) } \\
\text { bone anchors }(n=30)\end{array}$ & $\begin{array}{l}\text { Improvements seen in } \\
\text { both groups but no } \\
\text { statistical analysis from } \\
\text { baseline } \\
\text { measurements. } \\
3 \text { patient outcomes in } \\
\text { the no anchor group } \\
\text { regarded as clinical } \\
\text { failures. No failures in } \\
\text { suture anchor group. } \\
\text { Between group } \\
\text { improvements in MEPS } \\
\text { and DASH score in } \\
\text { favour of suture } \\
\text { anchor group at } 1,2,3, \\
6,12 \text { months } \\
\text { ( } p=0.001 \text { ). }\end{array}$ \\
\hline $\begin{array}{l}\text { Morgan et al } 2016^{19} \\
\text { RCT comparing platelet- } \\
\text { rich plasma injection vs } \\
\text { surgical release } \\
\text { Conducted in the UK }\end{array}$ & $\begin{array}{l}92 \text { patients but } 11 \\
\text { lost to follow up. } \\
\text { Mean age } 47 \\
34 \text { male, } 47 \text { female } \\
\text { Minimum symptom }\end{array}$ & $\begin{array}{l}\text { Platelet-rich plasma } \\
\text { (PRP) injection using a } \\
\text { peppering technique } \\
(n=42) \\
\text { Surgical release }(n=39)\end{array}$ & $\begin{array}{l}\text { 13/42 of PRP group } \\
\text { requested surgery } \\
\text { between } 2-6 \text { months } \\
\text { after injection } \\
1 / 39 \text { surgical patients } \\
\text { subsequently had a }\end{array}$ \\
\hline
\end{tabular}




\begin{tabular}{|c|c|c|c|}
\hline Conference abstract only & $\begin{array}{l}\text { duration } 6 \text { months } \\
\text { Failed non-operative } \\
\text { treatment }\end{array}$ & & $\begin{array}{l}\text { PRP injection } \\
\text { No significant } \\
\text { difference in Patient } \\
\text { Reported Tennis Elbow } \\
\text { Evaluation (PRTEE) or } \\
\text { DASH scores between } \\
\text { groups up to } 12 \\
\text { months. } \\
42 \% \text { of PRP patients } \\
\text { reported definite } \\
\text { benefit. }\end{array}$ \\
\hline $\begin{array}{l}\text { Radwan et al } 2008^{20} \\
\text { RCT comparing } \\
\text { extracorporeal } \\
\text { shockwave therapy } \\
\text { (ESWT) vs percutaneous } \\
\text { tenotomy } \\
\text { Conducted in Egypt }\end{array}$ & $\begin{array}{l}62 \text { patients but } 6 \\
\text { lost to follow up. } \\
\text { ESWT group: mean } \\
\text { age } 40.14(23-60) ; \\
15 \text { male, } 14 \text { female. } \\
\text { Tenotomy group: } \\
\text { mean age } 39.26 \text { ( } 22- \\
59) ; 18 \text { male, } 9 \\
\text { female } \\
\text { Minimum symptom } \\
\text { duration } 6 \text { months } \\
\text { with failed } \\
\text { conservative } \\
\text { treatment including } \\
\text { physiotherapy and } \\
\text { steroid injection }\end{array}$ & $\begin{array}{l}\text { ESWT to the common } \\
\text { extensor origin at the } \\
\text { point of maximum pain } \\
\text { with a dose of } 324.25 \\
\text { joules ( } n=29 \text { ) } \\
\text { Grundberg \& Dobson } \\
\text { technique }{ }^{27} \text { of } \\
\text { percutaneous common } \\
\text { extensor origin release } \\
\text { with back-slab plaster } \\
\text { immobilisation for } 1 \\
\text { week ( } n=27 \text { ) }\end{array}$ & $\begin{array}{l}\text { No significant } \\
\text { differences between } \\
\text { groups in any } \\
\text { measures at any time } \\
\text { up to } 12 \text { months. } \\
\text { VAS improved ( } p<0.01 \text { ) } \\
\text { at all time points in } \\
\text { both groups for } \\
\text { pressure pain up to } 12 \\
\text { months. } \\
\text { VAS improved ( } p<0.01 \text { ) } \\
\text { at } 3 \text { and } 6 \text { weeks in } \\
\text { both groups for rest } \\
\text { pain. } \\
\text { VAS improved ( } p<0.01 \text { ) } \\
\text { for night pain in ESWT } \\
\text { group up to } 12 \text { months } \\
\text { and tenotomy group } \\
\text { up to } 12 \text { weeks. }\end{array}$ \\
\hline $\begin{array}{l}\text { Yan et al } 2009^{21} \\
\text { RCT comparing open vs } \\
\text { arthroscopic Nirschl } \\
\text { release }^{23} \\
\text { Conducted in China } \\
\text { English abstract only }\end{array}$ & $\begin{array}{l}26 \text { patients ( } 28 \\
\text { elbows) } \\
\text { Mean duration of } \\
\text { conservative care } 23 \\
\text { months (4-60) }\end{array}$ & $\begin{array}{l}\text { Open Nirschl release }{ }^{23} \\
(n=13) \\
\text { Arthroscopic Nirschl } \\
\text { release }(n=15)\end{array}$ & $\begin{array}{l}\text { Mean follow up } 17.4 \\
\text { months (4-32) } \\
\text { No difference between } \\
\text { groups in pain VAS at } \\
\text { rest or with daily living, } \\
\text { return to work/sport } \\
\text { or satisfaction. }\end{array}$ \\
\hline
\end{tabular}




\begin{tabular}{|l|l|l|}
\hline $\begin{array}{l}\text { Full text only available in } \\
\text { Chinese language }\end{array}$ & & $\begin{array}{l}\text { Significant difference } \\
\text { in pain VAS at work \& } \\
\text { sports and MEPS in } \\
\text { favour of open group. } \\
\text { Open group: } 100 \% \\
\text { good or excellent } \\
\text { results. }\end{array}$ \\
& $\begin{array}{l}\text { Arthroscopic group: } \\
\text { 93.3 good or excellent } \\
\text { results. }\end{array}$ \\
& & \\
\hline
\end{tabular}

\section{Risk of Bias Assessment:}

Risk of bias assessment was conducted independently by two reviewers (MB \& $C L$ ) using the Cochrane Risk of Bias Assessment Tool. ${ }^{28}$ The process rates each study in terms of high risk, unclear risk and low risk of bias within seven domains based on published guidance. ${ }^{28}$ Any discrepancies between reviewers were then discussed and resolved. A third reviewer (AT) was available to cast a decisive vote, however this was not required. The outcomes were compiled using Review Manager (RevMan) [Computer program]. Version 5.3. Copenhagen: The Nordic Cochrane Centre, The Cochrane Collaboration, 2014; and used to generate the risk of bias charts (Figures $4 \& 5$ ).

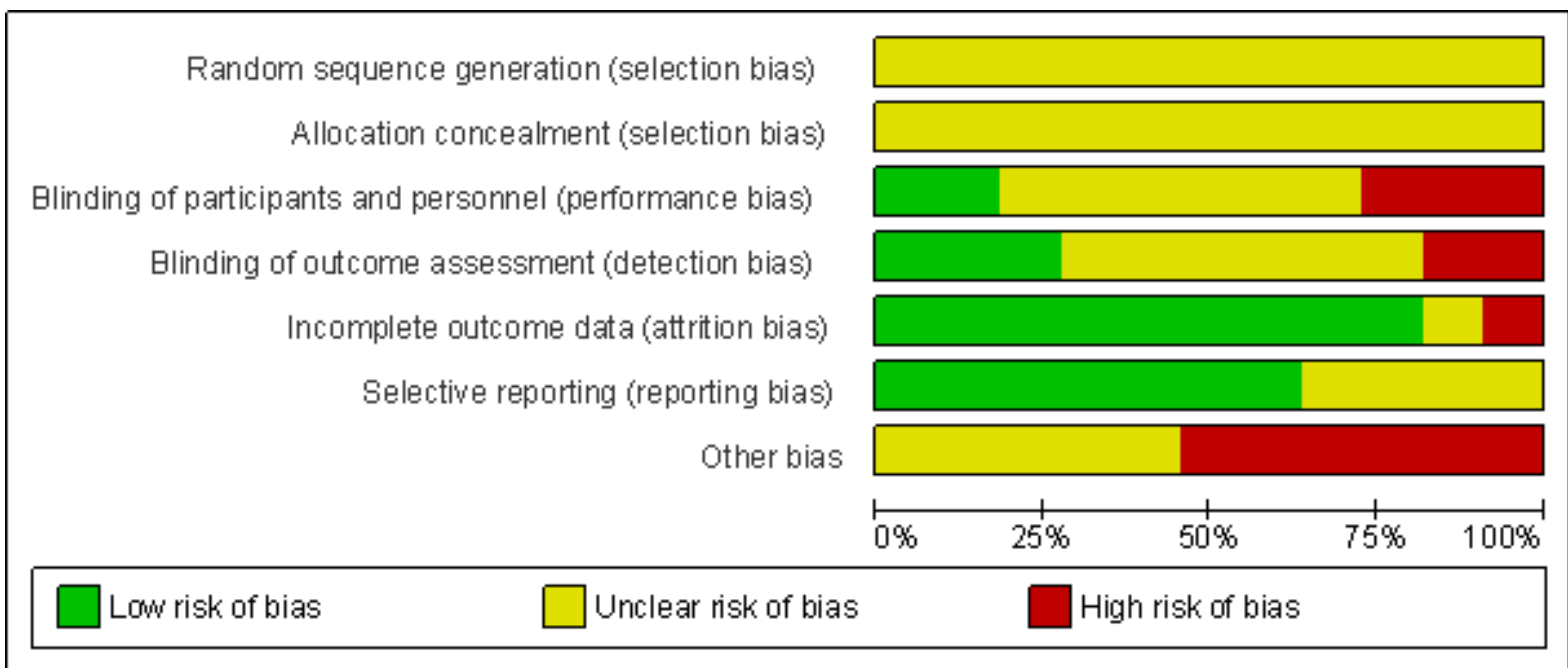

[Figure 4: Risk of bias across domains] 


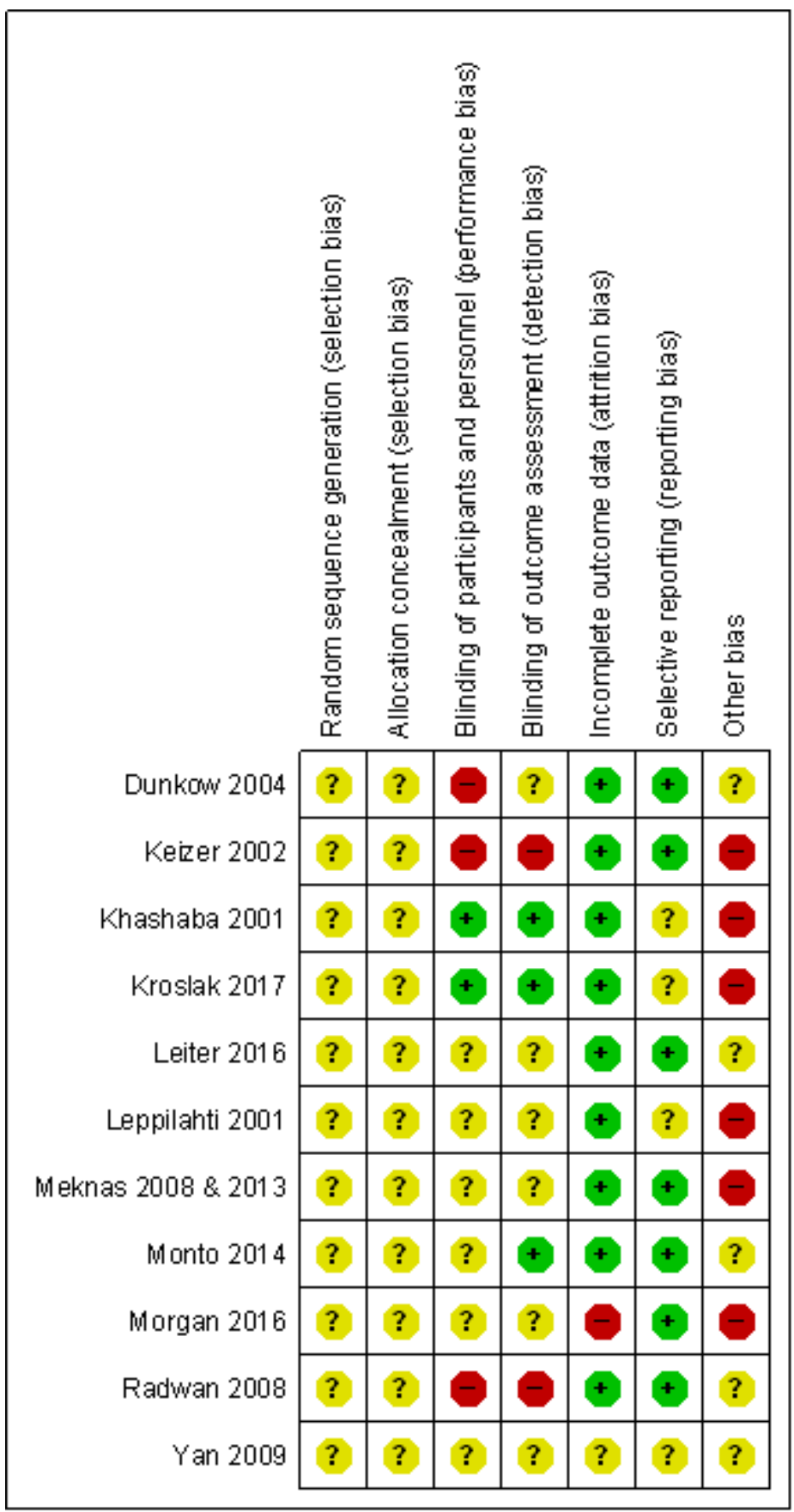

[Figure 5: Risk of bias per study]

Due to heterogeneity across the retrieved studies in relation to surgical interventions and measures of clinical outcome, a narrative synthesis based on levels of evidence was undertaken. ${ }^{29}$ This rating system, displayed in table 2 , is used to summarise the results in which the quality and outcomes of individual studies are taken into account. 


\begin{tabular}{|l|l|}
\hline Strong Evidence & Consistent findings in multiple high quality RCTs $(n>2)$ \\
\hline Moderate Evidence & Consistent findings among multiple lower quality RCTs and/ or 1 higher quality RCT \\
\hline Limited Evidence & Only one relevant low quality RCT \\
\hline Conflicting evidence & Inconsistent findings amongst multiple RCTs \\
\hline No evidence from trials & No RCTs \\
\hline
\end{tabular}

Table 2: Levels of Evidence

\section{RESULTS:}

124 abstracts were identified using database searches plus one additional paper from hand searching. After screening out duplicates and those that did not meet the inclusion criteria, 13 abstracts remained. Nine of these were available in full text versions plus three as conference abstracts only and one with an English translation abstract but full text in Chinese language. One full text was excluded as it was a protocol for a randomised controlled trial that had not yet been completed. ${ }^{22}$

The remaining 12 studies were assessed for risk of bias and the results are shown in figures $4 \& 5$. The two studies by Meknas et al ${ }^{16,17}$ investigated the same cohort of patients over different time points so details from each paper were merged for the assessment. It is notable that the risk of bias in all studies was unclear-to-high in five out of seven domains.

In total, the included studies investigated 490 patients (501 elbows). Four studies compared a surgical intervention versus non-surgical intervention (including sham surgery). ${ }^{11,13,19,20}$ We acknowledge that sham surgery still involves a surgical SKIN incision however we regard this as a non-surgical intervention or placebo as the area of pathology (i.e. the extensor tendons) are subject to no direct intervention and may not produce the same physiological changes. Two studies compared open versus arthroscopic surgical release. ${ }^{14,21}$ Two studies of the same patient group over different time periods investigated radiofrequency microtenotomy versus open release. ${ }^{16,17}$ Two studies compared the Nirschl surgical technique ${ }^{23}$ versus a modified technique. ${ }^{12,18}$ The remaining single studies compared open release versus a percutaneous mini-open technique; ${ }^{10}$ and open release versus posterior interosseous nerve decompression. ${ }^{15}$ In studies where interventions were similar, heterogeneity in the terms of outcome measures precluded synthesis using a meta-analysis.

There is moderate evidence (four relevant low quality RCTs) that surgery, such as the Nirschl technique of open release ${ }^{23}$, is not superior to non-surgical interventions, including Botox injection, shockwave therapy, platelet-rich plasma injection and sham surgery, up to 12 months in terms of the primary outcome measure of pain and function including subjective 
pain measures, modified Verhaar score, PRTEE and DASH scores. ${ }^{11,13,19,20}$ The sham surgery trial ${ }^{13}$ was terminated prematurely as a result.

There is conflicting evidence (inconsistent findings amongst two RCTs) in relation to the effectiveness of open versus arthroscopic surgical release. ${ }^{14,21}$ One RCT found no significant differences between groups in VAS, DASH score or grip power at any time up to 12 months $^{14}$ and the other RCT reported in favour of open surgery based on Mayo Elbow Performance Score and a sub-set of outcomes measuring pain VAS at work and during sport. $^{21}$

There is limited evidence (one relevant low quality RCT) suggesting no difference between radiofrequency microtenotomy and open surgical release in the short, medium or long term in relation to pain and function. ${ }^{16,17}$

There is limited evidence (two low quality RCTs ${ }^{12,18}$ ) in relation to the effectiveness of the standard Nirschl release compared to a modified surgical technique. ${ }^{12,18}$ Both found in favour of the modified technique: one RCT reported in favour of ECRB tendon repair using suture anchors at 12 months ${ }^{18}$ and another in favour of not drilling the epicondylar bone at six months. ${ }^{12}$

There is limited evidence (one relevant low quality RCT) suggesting no difference between mini-open percutaneous release and traditional open release in terms of DASH score at 12 months but a faster return to activity in the minimally invasive group was reported with median return to work of five weeks in the open group compared to 2 weeks in the percutaneous group $(p=0.0001) .^{10}$

There is limited evidence (one relevant low quality RCT) suggesting no difference between traditional open release and posterior interosseous nerve decompression at mean follow up of 31 months in terms of pain, grip strength and revision surgery. ${ }^{15}$

\section{DISCUSSION:}

The findings of this systematic review suggest that surgical interventions for tennis elbow are not more effective than non-surgical and sham interventions. Procedural modifications may enhance the comparative effectiveness of surgical interventions but have not been compared against placebo interventions. These findings, however, are based on a body of evidence with significant methodological limitations.

In keeping with previous systematic reviews ${ }^{7,8}$ these findings raise questions in relation to the effectiveness of surgery for tennis elbow and, considering risks and costs, whether nonsurgical interventions might be the current treatment of choice for this disorder. The question of how best to manage patients with persistent symptoms despite a period of failed conservative treatment still remains. Surgery has traditionally been regarded as being 
at the top of the treatment hierarchy but our findings suggest that it may not be any more effective than a further course of non-surgical treatment. However, this review also highlights the significant limitations in relation to the research evidence underpinning surgery for tennis elbow. Many of the included studies recruited small sample sizes with high likelihood of Type II error, had questionable or unclear methods of randomisation, allocation concealment and a lack of blinding of participants and outcome assessment. These significant methodological limitations give rise to a high risk of bias in the studies completed to date. Furthermore, there was a wide variation in the methods of outcome assessment used meaning that meaningful data synthesis, that might counteract some of the limitations of the individual trials, is compromised. Patient expectations have also not been considered in this study and evidence from rotator cuff surgery suggests that patient expectation of a surgical solution is the greatest predictor of whether conservative management fails. ${ }^{30}$

There seems adequate justification to propose further research in light of the significant methodological limitations of the current body of evidence, given the recalcitrant nature of tennis elbow for some patients and recognising that many do not resolve adequately with current treatment interventions. There is a clear indication for a high-quality, adequately powered RCT comparing surgical to sham surgical intervention including validated measures of patient reported pain and function. Unfortunately the reported sham study was terminated early due to a lack of difference in outcomes between groups but the small numbers of recruits mean that this was underpowered and mean that the conclusion that surgery is no more effective than placebo cannot be strongly supported. ${ }^{13}$ Despite the challenges associated with sham-controlled surgical trials, ${ }^{31}$ precedent has been set in the upper limb including a sham-controlled surgical trial investigating superior labral tears in the shoulder $^{32}$ and a current UK trial comparing arthroscopic shoulder decompression surgery to a sham procedure ${ }^{33}$, thus highlighting the feasibility of a larger trial.

\section{CONCLUSIONS:}

The findings of this systematic review suggest that surgical interventions for tennis elbow are not more effective than non-surgical or sham interventions for patients who have already undergone a course of conservative treatment. Caution is warranted though because these findings are based on a body of evidence with significant methodological limitations. There is now a clear indication for a high-quality, adequately powered RCT comparing surgical to sham surgical intervention, including validated measures of patient reported pain and function to inform future clinical practice. The inclusion of a third treatment arm in the form of a credible conservative intervention, e.g. a structured physiotherapy package or a wait-and-see approach, would allow conclusions to be drawn regarding the most efficient and effective treatment strategy. 
Funding: No funding received

Competing interests: None declared

\section{References:}

1. Bisset LM \& Vincenzino B. Physiotherapy management of lateral epicondylalgia. J. Physiother 2015;61:174-181.

2. Walker-Bone K, Cooper C. Hard work never hurt anyone-or did it? A review of occupational associations with soft tissue musculoskeletal disorders of the neck and upper limb. Ann Rheum Dis 2005;64:1112-17.

3. Harrington JM, Carter JT, Birrell L, et al. Surveillance case definitions for work related upper limb pain syndromes. Occup Environ Med 1998;55:264-71.

4. Silverstein B, Viikari-Juntura E, Kalat J. Use of a prevention index to identify industries at high risk for work-related musculoskeletal disorders of the neck, back, and upper extremity in Washington State, 1990-1998. Am J Ind Med. 2002;41:149-169.

5. Coombes BK, Bisset L, Vincenzino B. Management of Lateral Elbow Tendinopathy: One Size Does Not Fit All. J. Orthop. Sports Phys. Ther. 2015; 45(11):938-949.

6. Sanders TL Jr, Maradit Kremers H, Bryan AJ, et al. The epidemiology and health care burden of tennis elbow: a population-based study. Am J Sports Med 2015;43(5):1066-71.

7. Buchbinder R, Green S, Bell S et al. Surgery for lateral elbow pain. Cochrane Database Syst Rev. 2002;(1):CD003525.

8. Buchbinder R, Johnston RV, Barnsley L, et al. Surgery for lateral elbow pain. Cochrane Database Syst Rev 2011;(1):CD003525.

9. Moher D, Shamseer L, Clarke M, et al. Preferred Reporting Items for Systematic Review and Meta-Analysis Protocols (PRISMA-P) 2015 statement. Syst Rev. 2015;4(1):1.

10. Dunkow PD, Jatti M, Muddu BN. A comparison of open and percutaneous techniques in the surgical treatment of tennis elbow. J Bone J Surg [Br] 2004; 86-b (5):701-704.

11. Keizer SB, Rutten HP, Pilot $P$, et al. Botulinum toxin injection versus surgical treatment for tennis elbow. Clin Orthop Relat Res 2002;401:125-131. 
12. Khasaba A. Nirschl tennis elbow release with or without drilling. Br J Sports Med 2001;35:200-201.

13. Kroslak M \& Murrell G. Surgical treatment of lateral epicondylitis: A prospective, randomised, double blinded, placebo controlled clinical trial. I Sci Med Sport 2017;20S:e106-e128.

14. Leiter J, Clark T, McRae S, et al. Open versus arthroscopic tennis elbow release: randomized controlled trial. Orthop J Sports Med 2016;4(7)(suppl 4). DOI: $10.1177 / 2325967116 S 00167$.

15. Leppilahti J, Raatikainen T, Pienimäki T, et al. Surgical treatment of resistant tennis elbow. A prospective, randomised study comparing decompression of the posterior interosseous nerve and lengthening of the tendon of the extensor carpi radialis brevis muscle. Arch Orthop Trauma Surg 2001;121:329-332.

16. Meknas K, Odden-Miland A, Mercer JB, et al. Radiofrequency microtenotomy: a promising method for treatment of recalcitrant lateral epicondylitis. Am J Sports Med 2008;36(10):1960-1965.

17. Meknas K, Al Hassoni TN, Odden-Miland A, et al. Medium-term results after treatment of recalcitrant lateral epicondylitis: A prospective, randomized study comparing open release and radiofrequency microtenotomy. Orthop J Sports Med 2013;1(4):1-7.

18. Monto RR. Tennis elbow repair with or without suture anchors: a randomized clinical trial. Tech Should Surg 2014;15:92-97.

19. Morgan B , Trail IA, Watts AC, et al. Comparing platelet-rich plasma (PRP) injection with surgical intervention for the management of refractory lateral epicondylosis. a randomised clinical trial. Shoulder \& Elbow 2016;8(S1):S7-S28.

20. Radwan YA, ElSobhi G, Badawy WS, et al. Resistant tennis elbow: shock-wave therapy versus percutaneous tenotomy. Int Orthop (SICOT) 2008;32:671-677.

21. Yan H, Cui G-Q, Liu Y-L, et al. A randomized comparison of open and arthroscopic Nirschl debridement for refractory lateral epicondylitis. Zhonghua wai ke za zhi [Chinese journal of surgery] 2009;47(12):888-891.

22. Matache BA, Berdusco R, Momoli F, et al. A randomized, double-blind sham controlled trial on the efficacy of arthroscopic tennis elbow release for the management of chronic lateral epicondylitis. BMC Musc Dis 2016;17:239.

23. Nirschl RP. Lateral extensor release for tennis elbow. J Bone Joint Surg [Am]1994;76A:951. 
24. Hohmann G. Das wesen und die behandlung des sogenannten tennisellenbogens. munch med wochenschr 1933; 80:250-252.

25. Verhaar JA, Walenkamp G, Kester A, et al. Lateral extensor release for tennis elbow: A prospective long-term follow-up study. J Bone Joint Surg 1993;75A:1034-1043.

26. Paoloni JA, Appleyard RC, Murrell GAC. The Orthopaedic Research Institute-Tennis Elbow Testing System: a modified chair pick-up test-interrater and intrarater reliability testing and validity for monitoring lateral epicondylosis. J Should Elb Surg 2004;13(1):7277.

27. Grundberg AB, Dobson JF. Percutaneous release of the common extensor origin for tennis elbow. Clin Orthop 2000;376:137-140.

28. Higgins JPT, Green S (editors). Cochrane Handbook for Systematic Reviews of Interventions Version 5.1.0 [updated March 2011]. The Cochrane Collaboration, 2011. Available from www.handbook.cochrane.org.

29. van Tulder M, Furlan A, Bombardier C, et al. Updated Method Guidelines for Systematic Reviews in the Cochrane Collaboration Back Review Group. Spine 2003;28(12):12901299.

30. Dunn WR, Kuhn JE, Sanders R, et al. 2013 Neer Award: predictors of failure of nonoperative treatment of chronic, symptomatic, full-thickness rotator cuff tears. $J$ Shoulder Elbow Surg. 2016; 25: 1303-11.

31. Wartolowska K, Collins GS, Hopewell S, et al. Feasibility of surgical randomised controlled trials with a placebo arm: a systematic review. BMJ Open published online first 15th March 2016. doi:10.1136/bmjopen-2015-010194.

32. Schrøder CP, Skare $O$, Reikerås $O$ et al. Sham surgery versus labral repair or biceps tenodesis for type II SLAP lesions of the shoulder: a three-armed randomised clinical trial. Br J Sports Med published online first $12^{\text {th }}$ May 2017.

http://dx.doi.org/10.1136/bjsports-2016-097098.

33. Beard D, Jonathan Rees, Rombach I et al. The CSAW Study (Can Shoulder Arthroscopy Work?) - a placebo-controlled surgical intervention trial assessing the clinical and cost effectiveness of arthroscopic subacromial decompression for shoulder pain: study protocol for a randomised controlled trial. Trials published online $9^{\text {th }}$ May 2015. doi: 10.1186/s13063-015-0725-y 